\title{
Doses de cálcio no crescimento do feijoeiro cultivado em solução nutritiva, na presença de alumínio
}

\author{
Doses of calcium in the growth of the bean plant cultivated \\ in nutritive solution, in the presence of aluminum \\ Samuel de Assis Silva ${ }^{1}$, Willian Bucker Moraes ${ }^{2}$, Gustavo Soares de Souza ${ }^{3}$
}

\begin{abstract}
RESUMO
O objetivo deste trabalho foi o de observar os efeitos de níveis de cálcio no crescimento do feijoeiro (Phaseolus vulgaris L. cv. Talismã) cultivado em solução nutritiva, na presença do alumínio. O experimento foi conduzido em casa de vegetação, no Centro de Ciências Agrárias da Universidade Federal do Espírito Santo, localizado no município de Alegre-ES. As plantas de feijoeiro foram cultivadas durante 45 dias em solução nutritiva com doses crescentes de cálcio, nas concentrações de $0 ; 25 ; 50 ; 100$ e; $200 \mathrm{mg}$ $\mathrm{dm}^{-3}$ e dose única de alumínio a $15 \mathrm{mg} \mathrm{dm}^{-3}$. Utilizou-se o delineamento inteiramente casualizado, com cinco tratamentos e três repetições. O nível de $100 \mathrm{mg} \mathrm{dm}^{-3}$ de cálcio, favoreceu o desenvolvimento do sistema radicular e da parte aérea das plantas de feijoeiro inibindo a toxidez de alumínio.
\end{abstract}

Palavras-chave: Phaseolus vulgaris L., cálcio, toxidez de alumínio, análise de crescimento

\begin{abstract}
The objective of this study was to determine the effects of calcium on the growth of common bean plants (Phaseolus vulgaris L. cv. Talismã) in nutritive solution in the presence of aluminum. The experiment was performed in a greenhouse in the Centro de Ciências Agrárias da Universidade Federal do Espírito Santo in the municipal district of Alegre-ES. The bean plants were cultivated for 45 days in nutritive solution with increasing doses of calcium, in concentrations of 0,25, 50, 100 and $200 \mathrm{mg} \mathrm{dm}^{-3}$ and

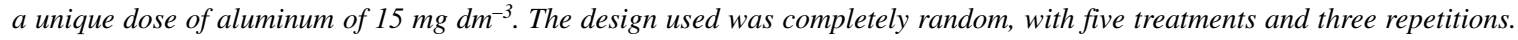
The level of $100 \mathrm{mg} \mathrm{dm}^{-3}$ calcium favored the development of the radicular system and the above-ground part of the bean plants, inhibiting the toxicity of the aluminum.
\end{abstract}

Key words: Phaseolus vulgaris L., calcium, aluminum toxicity, growth analysis.

\section{Introdução}

O feijoeiro comum é a espécie mais cultivada entre as demais do gênero Phaseolus, sendo considerado uma planta de grande importância para a alimentação da população brasileira. Esta cultura tem mostrado grandes flutuações quanto à produção, de ano para ano, pois seu cultivo, em grande parte, é conduzido em caráter secundário ou de subsistência (Hungria et al., 2000).

O feijoeiro é uma planta de ciclo curto, com pequeno e pouco profundo sistema radicular. Logo, é fundamental que os nutrientes sejam colocados à disposição da planta em quantidade, tempo e local adequado (Almeida et al., 2000). O cálcio é importante na preservação da capacidade de absorção das raízes mediante a manutenção da integridade da membrana plasmática, bem como na prevenção da perda de solutos para a solução externa, aumentando o acúmulo de nutrientes pela planta (Malavolta, 2006). A deficiência de cálcio, o aumento da acidez e o excesso de alumínio resultam em baixo crescimento e desenvolvimento do sistema radicular, com conseqüente exploração de menores volumes

1 Engenheiro Agrônomo, Mestre em Produção Vegetal, Doutorando em Engenharia Agrícola, Universidade Federal de Viçosa, Dept $^{\circ}$ de Engenharia Agrícola, Campus Universitário, CEP: 36570-000, Viçosa, Minas Gerais, Brasil.

E-mail: Samuel-assis@hotmail.com

2 Engenheiro Agrônomo, Mestre em Produção Vegetal, Doutorando em Proteção de Plantas, UNESP, Campus de Botucatu, Botucatu-SP, Brasil. E-mail: moraeswb@hotmail.com

3 Engenheiro Agrônomo, Mestre em Produção Vegetal, Doutorando em Engenharia Agrícola, UNICAMP, Faculdade de Engenharia Agrícola, Campinas-SP. E-mail: gsdsouza@hotmail.com

Fecha de Recepción: 25 Febrero, 2010.

Fecha de Aceptación: 15 Febrero, 2011. 
de solo, prejudicando a aborção de nutrientes e água, sujeitando as plantas à deficiências minerais e à défices hídricos (Lambais, 2006).

$\mathrm{O}$ excesso de alumínio inibe o crescimento e causa alterações na conformação do sistema radicular, tornando as raízes engrossadas, inchadas, com coloração marrom, menos ramificadas, quebradiças e com pontuações escuras nas extremidades (Epstein; Bloom, 2006). A ocorrência de toxicidade de alumínio em plantas cultivadas é freqüente em muitos solos brasileiros e, na maioria das vezes, está associada aos solos lixiviados, com baixa fertilidade e de elevada acidez (Veloso et al., 2000). Sabe-se que a acidez do solo tem grande importância na produtividade agrícola e nas práticas de manejo do solo, podendo de certa maneira, afetar diretamente o desenvolvimento e a constituição nutricional das plantas pela diminuição ou aumento da solubilidade de certos nutrientes, tornando-se necessário a sua correção através do uso de calcário (Silva et al., 2007), além disso os solos brasileiros são, em sua maioria, ácidos, e apresentam teores de alumínio em níveis fitotóxicos e baixos teores de cálcio e magnésio trocáveis, características desfavoráveis ao desenvolvimento da maioria das culturas (Sousa; Lobato, 2004).

O objetivo deste trabalho foi o de observar os efeitos de doses crescentes de cálcio em solução nutritiva, na presença de alumínio, no crescimento da parte aérea e do sistema radicular de feijoeiro comum.

\section{Material e Métodos}

O experimento foi conduzido em solução nutritiva, em casa de vegetação pertencente ao Centro de Ciências Agrárias da Universidade Federal do Espírito Santo, localizado no município de Alegre-ES a uma altitude de $150 \mathrm{~m}$, situado a $20^{\circ} 45^{\prime} \mathrm{S}$ e $41^{\circ}$ $29^{\prime}$ W, no período de outubro a dezembro de 2006. O clima da região, de acordo com a classificação de Köppen é do tipo "Aw", tropical, com médias de temperatura mínima e máxima anual, no local dos estudos, de $18^{\circ} \mathrm{C}$ e $31^{\circ} \mathrm{C}$, respectivamente.

As sementes de feijoeiro (Phaseolus vulgaris L. cv. Talismã), após tratamento com hipoclorito de sódio a $5 \%$, por um período de dois minutos, foram plantadas em recipientes plásticos contendo areia de rio lavada com água destilada e esterilizada. Após a emergência, aos doze dias após a semeadura, as plântulas foram cuidadosamente retiradas dos recipientes plásticos, sendo em seguida selecionadas, padronizando-se as mesmas quanto altura, número de folhas, quantidade das raízes e aspecto geral. A seguir, as raízes foram lavadas com água de torneira e imersas em água desmineralizada para completar a limpeza. Feito isso, as plântulas foram transferidas para recipientes plásticos com capacidade aproximada de 1,5 litros, contendo solução nutritiva, cujos tratamentos consistiram na aplicação de diferentes doses de cálcio, nas concentrações de $0 ; 25 ; 50$; 100 e $200 \mathrm{mg} \mathrm{dm}^{-3}$, adicionado como CaCO3. O alumínio foi adicionado em dose única de $15 \mathrm{mg}$ $\mathrm{dm}^{-3}$ para todos os tratamentos, na formulação Al2 (SO4)3 16 H2O-1M.

A composição química da solução nutritiva usada para o estudo dos efeitos das doses de cálcio no crescimento do feijoeiro na presença do alumínio baseou-se na solução completa apresentada por Hoagland e Arnon (1938), utilizando-se $1 / 2$ força, adaptada às condições de estudo. As soluções nutritivas, continuamente arejadas, foram renovadas a cada semana e o seu volume completado com água destilada diariamente. $\mathrm{O} \mathrm{pH}$ das soluções foi mantido em 4,0 \pm 0,2 com adições de $\mathrm{HCl}$ ou $\mathrm{NaOH}$.

Transcorridos quarenta e cinco dias contados a partir do início do experimento, as plantas foram colhidas e separadas em folhas, caules e raízes. A seguir fizeram-se os contornos de cada folha, em cada repetição, em cada tratamento, em papel heliográfico, para a determinação da área foliar (Benincasa, 2003). As partes aéreas e as raízes foram acondicionadas em sacos de papel e transferidas para estufa de circulação forçada de ar, a $70{ }^{\circ} \mathrm{C}$, até peso constante, para as determinações da massa da matéria seca. Foram feitas avaliações da Razão de Área Foliar \{RAF = área foliar $\left(\mathrm{m}^{2}\right)$ / matéria seca total (g) \}, Razão de Peso Foliar \{RPF $=$ matéria seca de folhas $(\mathrm{g}) /$ matéria seca total $(\mathrm{g})\}$, Área Foliar Específica $\left\{\mathrm{AFE}=\right.$ área foliar $\left(\mathrm{m}^{2}\right)$ / matéria seca de folhas (g)\}, Taxa de Assimilação Líquida $\{\mathrm{TAL}=[($ matéria seca total $2-$ matéria seca total 1) / (área foliar 2 - área foliar 1) / 2]\} / intervalo de tempo entre as coletas em dias\}, Taxa de Crescimento Absoluto $\{\mathrm{TCA}=$ [matéria seca total 2 - matéria seca total 1 / intervalo de tempo entre as coletas em dias]\} e Taxa de Crescimento Relativo $\{$ TCR $=[$ RAF x TAL $]\}$ (Benincasa, 2003). Avaliou-se também o aparecimento de sintomas visuais de toxidez nas plantas. 
O delineamento experimental utilizado foi o inteiramente casualizado, com cinco tratamentos e três repetições, sendo uma planta por repetição.

Os resultados do experimento foram submetidos à análise de variância, sendo as médias comparadas por intermédio do teste de Tukey, a 5\% de probabilidade. As análises foram realizadas utilizando-se o pacote estatístico Statistica 6.0 (2001).

\section{Resultados e Discussão}

A massa de matéria seca, tanto na parte aérea quanto no sistema radicular das plantas, foi significativamente maior no nível $100 \mathrm{mg} \mathrm{dm}^{-3}$ de cálcio, decrescendo quando a concentração foi de $200 \mathrm{mg}$ $\mathrm{dm}^{-3}$ (Tabela 1).

Sabe-se que o cálcio exerce diversas funções nas plantas, incluindo a estabilização da membrana plasmática, faz parte da lamela média e está associado na transdução de sinais nas células (Taiz; Zeiger, 2004). Silva et al. (1993), trabalhando em solo e com diferentes localizações de $\mathrm{CaCO} 3$ e $\mathrm{P}$ em vasos geminados, também verificaram que a aplicação de Ca favoreceu o desenvolvimento do sistema radicular, em particular das raízes finas em plantas de milho. O nível de $100 \mathrm{mg} \mathrm{dm}^{-3}$ de cálcio corresponde aproximadamente à metade da concentração desse elemento na solução completa de Hoagland e Arnon, indicando que $200 \mathrm{mg} \mathrm{dm}^{-}$ ${ }^{3}$ causa decréscimos no crescimento, provavelmente
Tabela 1. Massa de matéria seca da parte aérea (PA) e do sistema radicular (SR) em feijoeiros cultivados por 45 dias em solução nutritiva contendo doses crescentes de cálcio e dose única de alumínio.

\begin{tabular}{lccccc}
\hline \multirow{2}{*}{ Parte da planta } & \multicolumn{5}{c}{ Níveis de cálcio $\left(\mathrm{mg} \mathrm{dm}^{-3}\right)$} \\
\cline { 2 - 6 } & 0 & 25 & 50 & 100 & 200 \\
\hline PA $(\mathrm{g})$ & $0,14 \mathrm{c}^{(1)}$ & $0,76 \mathrm{~b}$ & $0,66 \mathrm{~b}$ & $2,18 \mathrm{a}$ & $1,52 \mathrm{~b}$ \\
SR $(\mathrm{g})$ & $0,05 \mathrm{~d}$ & $0,54 \mathrm{~b}$ & $0,38 \mathrm{c}$ & $0,97 \mathrm{a}$ & $0,88 \mathrm{~b}$ \\
\hline
\end{tabular}

(1) Médias seguidas por letras diferentes, na mesma linha, diferem significativamente entre si pelo teste de Tukey a $5 \%$ de probabilidade.

competindo na absorção e utilização de outros elementos essenciais.

Com o aumento dos níveis de cálcio, foi observado um comportamento polinomial tanto da matéria seca de parte aérea, quanto do sistema radicular, conforme observado na Figura 1.

Os menores crescimentos ocorreram na ausência de cálcio na solução nutritiva, sendo que os níveis de 25 e $50 \mathrm{mg} \mathrm{dm}^{-3}$ de cálcio também limitaram o crescimento, por estarem em níveis baixos, não garantindo um suprimento adequado das plantas, e/ ou os baixos níveis do cálcio não foram suficientes para minimizar os efeitos nocivos do alumínio. Os níveis de $\mathrm{Al}$ utilizados neste trabalho $\left(15 \mathrm{mg} \mathrm{dm}^{-3}\right)$ foram considerados nocivos para as plantas, uma vez que para outras leguminosas, como exemplo, Stylosanthes guianensis, $9 \mathrm{mg} \mathrm{dm}^{-3} \mathrm{em}$ solução

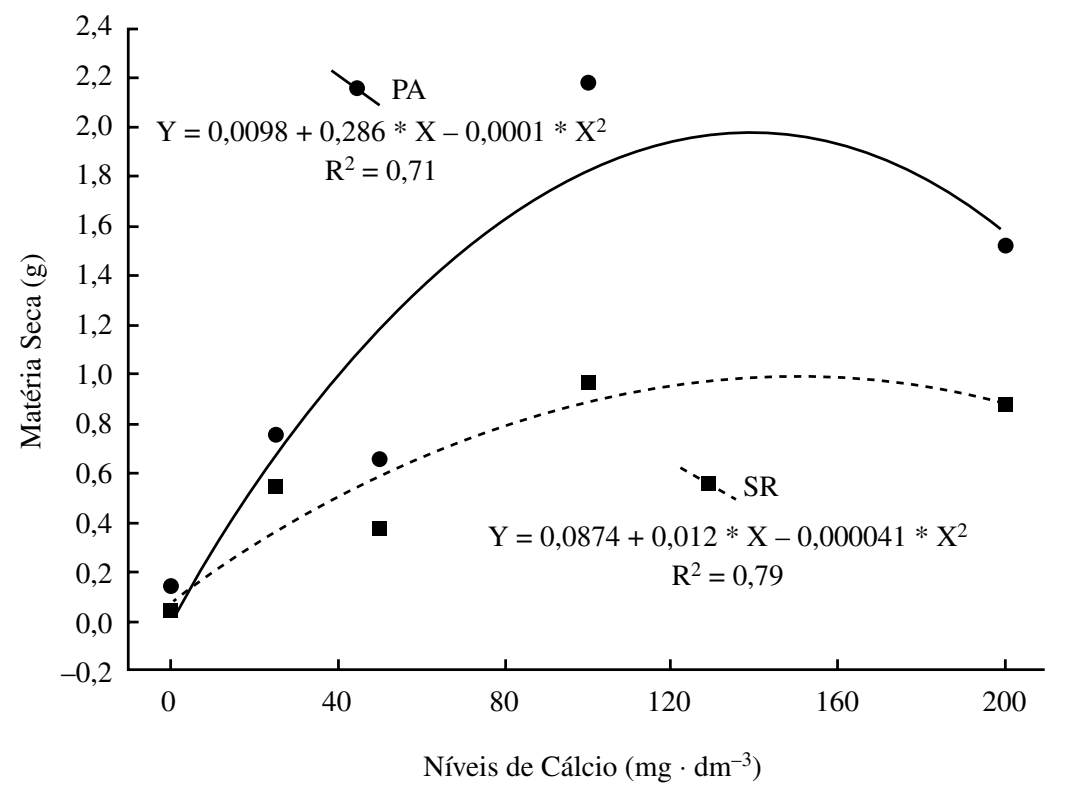

Figura 1. Matéria seca de parte aérea (PA) e sistema radicular (SR) de plantas de feijoeiro em função de níveis crescentes de cálcio na presença de alumínio. 
nutritiva, por doze dias de cultivo, causaram toxidez nas plantas (Amaral et al., 2000). Nolla et al. (2007) observaram que o alumínio reduz consideravelmente o crescimento radicular das plântulas de leguminosas além de aumentar a espessura das raízes, inibindo a expansão das células da raiz, culminando no seu engrossamento.

O cálcio contribui para o crescimento e multiplicação das raízes das plantas (Souza et al. 2000), sendo que o aumento da sua concentração no ambiente radicular pode diminuir, por competição, a adsorção do $\mathrm{Al}$ à plasmalema das células, e ter funções metabólicas específicas que minimizem os efeitos danosos do $\mathrm{Al}$, como, por exemplo, a estabilização da membrana plasmática pelo Ca (Malavolta, 2006), além disso o aumento da concentração de $\mathrm{Ca}$ em solução diminui o efeito tóxico do Al, por reduzir a atividade do $\mathrm{Al}+$ na superfície externa da membrana plasmática das células da raiz (Silva et al., 2005).

Mattiello et al. (2008), estudando o efeito do Al na cultura do cafeeiro, observou que esse elemento prejudicou o desenvolvimento das plantas devido ao seu efeito tóxico além de reduzir o potencial das plantas de absorver outros nutrientes como fósforo.

Os dados referentes ao efeito dos tratamentos das avaliações das plantas de P. vulgaris L. é apresentado na Tabela 2.

A área foliar apresentou melhor desenvolvimento nos tratamentos com as concentrações de $100,200,25$ e $50 \mathrm{mg} \mathrm{dm}^{-3}$, respectivamente. O tratamento com ausência de cálcio apresentou os menores valores. Resultados semelhantes foram observados por Rodrigues et al. (1993) trabalhando com Stylosanthes guyanensis. O cálcio é um mineral fundamental para a obtenção de plantas dotadas de grande área foliar, estabelecendo condições para alta atividade fotossintética, visando à produção de massa foliar. Isso indica que na carência desse nutriente há uma considerável redução na área foliar.

Ainda na Tabela 2, observa-se que não houve diferença significativa na RAF entre os tratamentos, o que indica que as doses de cálcio não influenciaram na conversão de matéria seca das plantas, uma vez que segundo Benincasa (2003) a RAF é a relação de quanto de área foliar é necessária para produzir um grama de matéria seca total. Tal comportamento pode ter sido uma resposta à quantidade excessiva de alumínio em solução nutritiva que afetou diretamente o sistema radicular das plantas. Resultados semelhantes foram observados por Veloso et al. (2000), em estudo com pimenta do reino.

Freitas et al. (2006), afirma que o excesso de $\mathrm{Al}$ nas plantas em geral interfere na divisão celular das raízes, aumenta a rigidez da parede celular pela ligação com as pectinas, reduz a produção de ácido desoxirribonucléico, diminui a respiração das raízes, interfere na ação das enzimas que governam a fosforilação de açúcares e precipita polissacarídeos da parede celular, além de perturbar a absorção, transporte e uso de vários nutrientes.

A AFE, que mede a densidade das folhas, apresentou maiores valores nos tratamentos com concentrações médias de cálcio $\left(50\right.$ e $25 \mathrm{mg} \mathrm{dm}^{-3}$ ), o que demonstra que algumas oscilações foram verificadas nesse parâmetro e não foram observadas na RAF, o que pode ser justificado, pois essas oscilações resultam das taxas de crescimento das folhas individuais. Segundo Zabot et al. (2004) com o avanço da idade da folha, ocorre aumento na produção e também maior demanda por fotoassimilados, ocasionando oscilações na AFE.

Tabela 2. Área foliar (AF), massa de matéria seca (MS), razão de peso foliar (RPF), Área foliar específica (AFE), taxa de crescimento absoluto (TCA), taxa de crescimento relativo (TCR), taxa assimilatória líquida (TAL) e razão de peso foliar (RPF) em feijoeiro cultivado em solução nutritiva contendo níveis crescentes de cálcio (NC) e dose única de alumínio.

\begin{tabular}{|c|c|c|c|c|c|c|c|c|}
\hline $\begin{array}{l}\mathrm{NC} \\
\left(\mathrm{mg} \cdot \mathrm{dm}^{-3}\right)\end{array}$ & $\begin{array}{c}\mathrm{AF} \\
\left(\mathrm{cm}^{2}\right)\end{array}$ & $\begin{array}{l}\text { MS } \\
(\mathrm{g})\end{array}$ & $\begin{array}{c}\mathrm{RPF} \\
\left(\mathrm{g} \mathrm{dia}^{-1}\right)\end{array}$ & $\begin{array}{c}\text { AFE } \\
\left(\mathrm{m}^{2} \mathrm{~g}^{-1}\right)\end{array}$ & $\begin{array}{c}\text { TCA } \\
\left(\mathrm{g} \mathrm{dia}^{-1}\right)\end{array}$ & $\begin{array}{c}\text { TCR } \\
\left(\mathrm{g} \mathrm{g}^{-1} \mathrm{dia}^{-1}\right)\end{array}$ & $\begin{array}{c}\text { TAL } \\
\left(\text { g.m } \mathrm{m}^{-2} \mathrm{dia}^{-1}\right)\end{array}$ & $\begin{array}{c}\text { RAF } \\
\left(\mathrm{m}^{2} \mathrm{~g}^{-1}\right)\end{array}$ \\
\hline 0 & $36,31 \mathrm{c}^{(1)}$ & $0,330 \mathrm{c}$ & $0,638 \mathrm{a}$ & $0,021 \mathrm{c}$ & $0,0003 \mathrm{~b}$ & $0,0298 \mathrm{c}$ & $0,1668 \mathrm{c}$ & $0,6584 \mathrm{a}$ \\
\hline 25 & $304,35 \mathrm{ab}$ & $2,059 \mathrm{~b}$ & $0,400 \mathrm{a}$ & $0,040 \mathrm{a}$ & $0,0387 \mathrm{~b}$ & $0,2891 \mathrm{~b}$ & $0,6736 \mathrm{~b}$ & $0,4403 \mathrm{a}$ \\
\hline 50 & $245,64 \mathrm{~b}$ & $1,699 \mathrm{c}$ & $0,752 \mathrm{a}$ & 0,041 a & $0,0307 \mathrm{~b}$ & $0,3405 \mathrm{~b}$ & $0,7038 \mathrm{~b}$ & $0,7928 \mathrm{a}$ \\
\hline 100 & $711,97 \mathrm{a}$ & $5,335 \mathrm{a}$ & 0,466 a & $0,039 \mathrm{~b}$ & $0,1115 \mathrm{a}$ & $0,3162 \mathrm{~b}$ & $0,7949 \mathrm{~b}$ & $0,5046 \mathrm{a}$ \\
\hline 200 & $495,66 \mathrm{a}$ & $3,916 \mathrm{a}$ & $0,761 \mathrm{a}$ & $0,021 \mathrm{~b}$ & $0,0799 \mathrm{a}$ & $0,8439 \mathrm{a}$ & $2,1682 \mathrm{a}$ & $0,7817 \mathrm{a}$ \\
\hline
\end{tabular}

(1) Médias seguidas por letras diferentes, na mesma coluna, diferem significativamente entre si pelo teste de Tukey a 5\% de probabilidade. 
A TCR, que representa o aumento em gramas de biomassa seca por unidade de material presente num período de observação, seguiu comportamento semelhante aos demais parâmetros. Observa-se que a TCR do tratamento com concentração de cálcio de $200 \mathrm{mg} \mathrm{dm}^{-3}$ foi maior que os demais tratamentos. A mesma tendência foi observada para a TAL, que é um componente da TCR, o que explica a mesma tendência. Os dados da TAL sugerem maior eficiência na produção de fotossintetizados por área de folha na concentração mais alta de cálcio em relação aos demais tratamentos, principalmente em relação àquele sem a presença do nutriente, conforme pode também ser observado na Figura 2.

O modelo estatístico de melhor ajuste (5\% de significância) para a relação Taxa de crescimento relativo (TCR) e Taxa de assimilação líquida (TAL) versus níveis de cálcio foi o polinomial do $2^{\circ}$ grau, como pode ser visto na Figura 2, sendo que $94 \%$ da TCR pode ser explicada pela equação $\mathrm{Y}=0,1257+0,0024 * \mathrm{X}+0,0000054 * \mathrm{X} 2$, enquanto que $95,8 \%$ da TAL é explicada pela equação $\mathrm{Y}=$ $0,3509+0,0035 * \mathrm{X}+0,000027 * \mathrm{X} 2$.

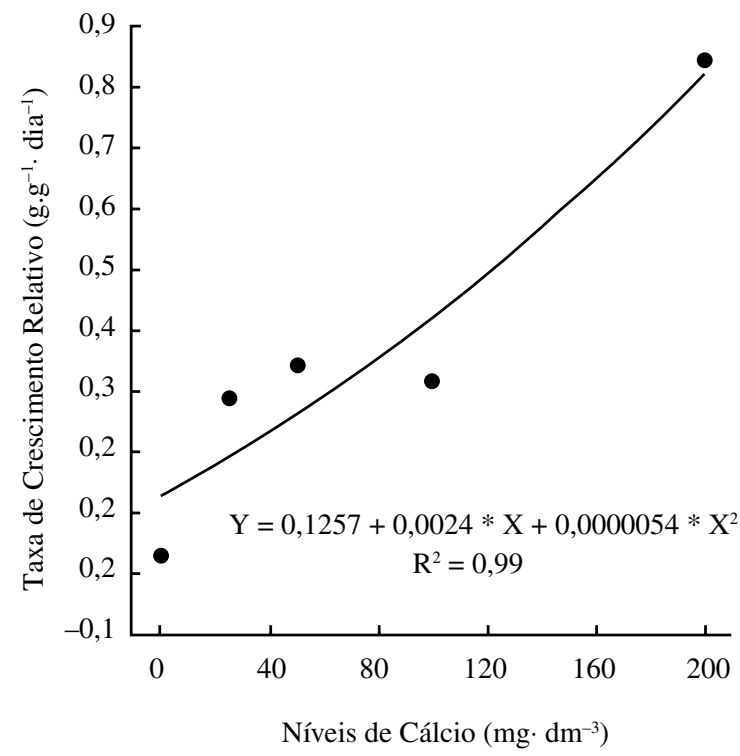

Tais valores de TCR e TAL indicam a existência de uma relação entre níveis de cálcio e eficiência da matéria vegetal em produzir matéria seca. Porém, segundo Caines \& Shennan (1999), a relação entre o uso eficiente de Ca e o crescimento de planta é muito complexa e pode envolver vários controles fisiológicos, como a capacidade de retranslocação interna de Ca compartimentalizado em membranas e órgãos celulares de armazenamento como retículo endoplasmático, cloroplastos e vacúolo. No entanto alguns autores como Rosolem et al. (2000), Wutke et al. (2003) e Silva et al. (2004), em seus estudos constataram maior crescimento e rendimento de cultivares de feijoeiro quando do suprimento de cálcio às plantas em comparação a tratamentos onde este nutriente foi omitido.

\section{Conclusões}

A concentração de $100 \mathrm{mg} \mathrm{dm}^{-3}$ de cálcio favorece o desenvolvimento do sistema radicular e da parte aérea das plantas do feijoeiro comum, influenciando na eficiência da matéria vegetal em produzir matéria seca e inibindo a toxidez de alumínio.



Figura 2. Taxa de crescimento relativo e Taxa de assimilação líquida de plantas de feijoeiro em função de níveis crescentes de cálcio na presença de alumínio 


\section{Referências Citadas}

Almeida, C. De; Carvalho, M.A.C. De; Arf, O.; Sá, M.E. De; Buzetti, S.

2000 Uréia em cobertura e via foliar em feijoeiro. Scientia Agricola, Piracicaba, v.57, n.2.

Amaral, J.A.T.; Cordeiro, A.T.; Rena, A.B.

2000 Efeitos do alumínio, nitrato e amônio sobre a composição de metabólitos nitrogenados e de carboidratos em Stylosanthes guianensis e S. macrocephala. Pesquisa Agropecuária Brasileira, v.35, n.2, p. 313-320.

Benincasa, M.M.P.

2003 Análise de crescimento de plantas (noções básicas). Jaboticabal-SP. Funep. 41 p.

Caines, A.M.; Shennan, C.

1999 Growth and nutrient composition of $\mathrm{Ca}^{2+}$ use efficient and $\mathrm{Ca} 2+$ use inefficient genotypes of tomato. Plant Physiology. Biochem, 37: 559-567.

Epstein, E.; Bloom, A.J.

2006 Nutrição mineral de plantas: princípios e perspectivas. Planta Editora. 2 ed. 403 p.

Freitas, F.A.; Kopp, M.M.; Sousa, R.O.; Zimmer, P.D. Carvalho, F.I.F. Oliveira, A.C.

2006 Absorção de P, Mg, Ca e K e tolerância de genótipos de arroz submetidos a estresse por alumínio em sistemas hidropônicos. Ciência Rural, v.36, n.1, jan-fev.

Hungria, M. Andrade, D.S., Chueire, L.A.O., Probanza, A., Guttierrez-Manero, F.J., Megias, M.

2000 Isolation and characterization of new efficient na competitive bean (Phaseolus vulgaris L.) rhizobia from Brazil Soil Biology \& Biochesmistry, v.32, p. 1515-1528.

Lambais, M.R.

2006 Unraveling the signaling and signal transduction mechanisms controlling arbuscular mycorrhiza development. Scientia Agricola. v.63, n.4, p. 405-413.

Malavolta, E.

2006 Manual de nutrição mineral de plantas. Editora Ceres. $638 \mathrm{p}$.

Mattiello, E.M.; Pereira, M.G.; Zonta, E.; Mauri, J.; Matiello, J.D.; Meireles, P.G.; Silva, I.R.

2008 Produção de matéria seca, crescimento radicular e absorção de cálcio, fósforo e alumínio por Coffea canephora e coffea arabica sob influência da atividade do alumínio em solução. Revista Brasileira de Ciência do Solo, 32: 425-434.

Moraes, J.F.L.; Bellingieri, P.A.; Fornasieri Filho, D.; Galon, J.A. 1998 Efeito de doses de calcário e de gesso na cultura do feijoeiro (Phaseolus vulgaris L.) cv. carioca-80. Scientia Agricola, Piracicaba, v.55, n.3.

Nolla, A.; Schlindwein, J.A.; Anghinoni, I.

2007 Crescimento, morfologia radicular e liberação de compostos orgânicos por plântulas de soja em função da atividade de alumínio na solução do solo de campo natural. Ciência Rural, v.37, n.1, jan-fev.

Rodrigues, J.D.; Rodrigues, S.D.; Pedras, J.F.; Delachiave, M.E.A.; Boaro, C.S.F.; Ono, E.O.

1993 Diferentes níveis de cálcio e o desenvolvimento de plantas de estilosantes (Stylosanthes guyanensis (aubl.)
SW. cv "Cook"). Scientia Agricola, Piracicaba, v.50, n.3, jan./set.

Rosolem, C.A.; Giommo, G.S.; Laurenti, R.L.B.

2000 Crescimento radicular e nutrição de cultivares de algodão em resposta a calagem. Pesquisa Agropecuária Brasileira, v.35, p. 827-833.

Silva, D.J.; Alvarenga, R.C.; Alvarez Venegas, V.H.; Soares, P.C. 1993 Localização de fósforo e de cálcio no solo e seus efeitos sobre o desenvolvimento inicial de milho. Revista Brasileira de Ciência do Solo, Campinas, SP, v.17, p. 203-209.

Silva, I.R.; Ferrufino, A.; Sanzonowicz, C.; Smyth, T. J.; Israel, D.W. \& Carter Junior, T.E.

2005 Interactions between magnesium, calcium and aluminum on soybean root elongation. Revista Brasileira de Ciência do Solo, 29: 747-754.

Silva, L.M.; Lemos, L.B.; Crusciol, C.A.C.; Feltran, J.C. 2004 Sistema radicular de cultivares de feijão em resposta à calagem. Pesquisa Agropecuária Brasileira, v.39, n.7 p. 701-707, jul.

Silva, M.A.C.; Natale, W.; Prado, R.M.; Corrêa, M.C.M.; Stuchi, E.S.; Andrioli, I.

2007 Aplicação superficial de calcário em pomar de laranjeira pêra em produção. Revista Brasileira de Fruticultura. v.29, n.3, p. 606-612.

Sousa, D.M.; Lobato, E. 2004 Cerrado: correção do solo e adubação. 2.ed. Brasília: Embrapa Informação Tecnológica, 416 p.

Souza, L.F. Da; Trindade, A.V.; Oliveira, A.M.G 2000 Calagem, exigências nutricionais e adubação. In: Mamão, produção: aspectos técnicos. Brasília: Embrapa Comunicação para Transferência de Tecnologia, p. 26-34.

Stat Soft Inc, 2001 Programa Statistica for windows, versão 6.0. Tulsa, EUA: Stat Soft, Inc, 1 CD-ROM.

Taiz, L.; Zeiger, E. 2004 Fisiologia vegetal. Porto Alegre: Artmed, p. 449-484.

Veloso, C.A.C.; Malavolta, E.; Muraoka, T.; Carvalho, E.J.M. 2000 Alumínio e a absorção de cálcio por mudas de pimenta do reino. Scientia Agricola, Piracicaba, v.57, n.1.

Wutke, E.B.; Pires, R.C.M.; Tanaka, R.T.; Sakai, E.; Mascarenhas, H.A.A.

2003 Desenvolvimento vegetativo e radicular, rendimento de grãos e qualidade fisiológica de sementes de feijoeiro da seca após cultivo de adubos verdes, em plantio direto. Revista de Agricultura, v.78, p. 77-91.

Zabot, L.; Dutra, L.M.C.; Jauer, A.; Lucca Filho, O.A.; Uhry, D.; Stefanel, C.; Losekan, M.E.; Farias, J.R.; Ludwig, M.P. 2004 Análise de crescimento da cultivar de feijão BR IPAGRO 44 guapo brilhante cultivada na safrinha em quatro densidades de semeadura em Santa Maria-RS. Revista de Ciências Agroveterinárias, Lages, v.3, n.2, p. 105-115. 